\title{
Computerized Detection of Masses in Digital Mammograms: Investigation of Feature-Analysis Techniques
}

\author{
Fang-Fang Yin, Maryellen L. Giger, Kunio Doi, Carl J. Vyborny, and Robert A. Schmidt
}

\begin{abstract}
Mammographic screening of asymptomatic women has shown effectiveness in the reduction of breast cancer mortality. We are developing a computerized scheme for the detection of mammographic masses as an aid to radiologists in mammographic screening programs. Possible masses on digitized screen/film mammograms are initially identified using a nonlinear bilateral-subtraction technique, which is based on asymmetric density patterns occurring in corresponding portions of right and left mammograms. In this study, we analyze the characteristics of actual masses and nonmass detections to develop feature-analysis techniques with which to reduce the number of nonmass (ie, false-positive) detections. These featureanalysis techniques involve (1) the extraction of various features (such as area, contrast, circularity and border-distance based on the density and geometric information of masses in both processed, and original breast images |, and (2) tests of the extracted features to reduce nonmass detections. Cumulative histograms of both actual-mass detections and nonmass detections are used to characterize extracted features and to determine the cutoff values used in the feature tests. The effectiveness of the feature-analysis techniques is evaluated in combination with the computerized detection scheme that uses the nonlinear bilateralsubtraction technique using free-response receiver operating characteristic analysis and 77 patient cases (308 mammograms). Results show that the featureanalysis techniques effectively improve the performance of the computerized detection scheme: about $35 \%$ false-positive detections were eliminated without loss in sensitivity when the feature-analysis techniques were used.

Copyright 1994 by W.B. Saunders Company
\end{abstract}

KEYWORDS: digital mammography, computer-vision, free-response receiver operating characteristic (FROC) analysis, feature analysis, computer-aided diagnosis.

$\mathbf{M}$ AMMOGRAPHIC screening of asymptomatic women has shown effectiveness in the reduction of breast cancer mortality. ${ }^{1,2}$

From the Kurt Rossmann Laboratories for Radiologic Image Research, Department of Radiology, The University of Chicago, Chicago, IL.

Supported in part by an American Cancer Society Faculty Research Award FRA-390, and USPHS Grant Nos. CA48985 and $C A 24806$

Address reprint requests to Maryellen L. Giger, PhD, Department of Radiology, MC2026, The University of Chicago, 5841 S Maryland Ave, Chicago, IL 60637.

Copyright $\odot 1994$ by W.B. Saunders Company

0897-1889/94/0701-0008\$03.00/0
However, this screening procedure generates a large volume of mammograms that must be interpreted by radiologists. To assist radiologists in such screening programs, we are developing a computerized scheme for the detection of masses in digital mammograms. ${ }^{3,4}$ Masses on mammograms can be difficult to detect, yet have higher positive biopsy yield of cancer than calcifications and other mammographic abnormalities, and are more likely to represent invasive cancer. Our computerized detection scheme involves: (1) the isolation of the breast region on the digital mammogram and the alignment of two corresponding breast images; (2) the identification of regions suspicious for possible masses; and (3) the feature analysis of suspicious regions to reduce the number of nonmass (falsepositive) detections. The initial identification of possible masses uses a nonlinear bilateralsubtraction technique. ${ }^{4}$

The visual features of regions identified as suspicious masses may be used as additional criteria for the differentiation between normal and abnormal tissues..$^{5-7}$ In this study, we analyzed by computer the features of actual-mass and nonmass detections identified initially using the nonlinear bilateral-subtraction technique as a basis for reducing the number of nonmass detections (ie, false-positive detections). Here, nonmass detections refer to those computerreported locations that correspond to areas of normal breast tissue. The feature-analysis techniques involve (1) the extraction of various features for each of identified suspicious regions on both processed and original images (such as area, contrast, circularity and borderdistance based on the density and geometric information of masses in both processed and original breast images) and (2) tests of the extracted features to classify each suspicious region as either "mass" or "nonmass." Cumulative histograms of both actual-mass detections and nonmass detections are used to optimize the feature-analysis techniques. The effectiveness of each feature-analysis technique is evaluated using free-response receiver operating characteristic $^{8,9}$ (FROC) analysis. 


\section{MATERIALS}

The database used in this study was obtained from clinical screen/film mammograms (Kodak MinR screen/OM-1 film; Rochester, NY). Each patient case includes two pairs of mammograms: right and left mediolateral oblique (MLO) views, and right and left craniocaudal views. The database was comprised of 77 clinical cases, totalling 154 pairs of mammograms ( 90 of which had one mass per pair). All masses present in the mammograms were verified from biopsy and pathology reports. These 90 masses were rated subjectively on basis of their conspicuity by an expert radiologist using a fivecategory scale as shown in Fig 1 . The database has been described elsewhere in detail with respect to subjectivity, lesion size and lesion contrast. ${ }^{4,10}$ it should be noted that most masses are in the three subtle categories.

The screen/film mammograms were digitized using a laser film digitizer (Konica KFRD-S) with a sampling distance of $.131 \mathrm{~mm}$ and 10-bit quantization. Digitized mammograms were then subsampled to form $512 \times 512$ matrices, having an effective sampling distance of $.4 \mathrm{~mm}$. Figure 2 shows an example of a pair of digitized mammograms obtained in the MLO view, with a malignant mass indicated by an arrow in the right breast image.

\section{METHODS}

\section{Computerized Detection Scheme}

The computerized scheme for the detection of masses was developed to extract automatically the differences in radiographic appear-

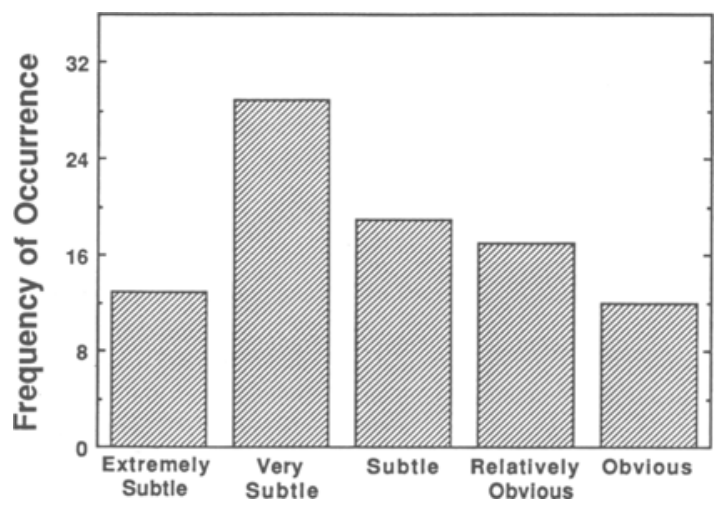

Fig 1. Subtlefy distribution of masses. A total of 90 masses were subjectively rated by an expert radiologist into five categories.

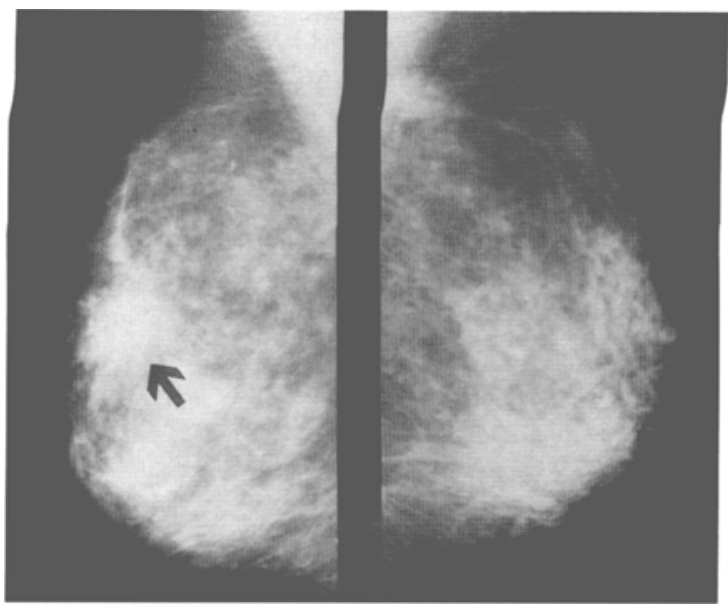

Fig 2. Example of a digitized mammogram in MLO view, with a malignant mass indicated by an arrow.

ances of masses and normal tissues in mammograms. Mammographic masses in screen/film mammograms usually are identified visually using criteria ${ }^{5-7}$ of density, geometric pattern, and asymmetry between the right and left breasts. The nonlinear bilateral-subtraction technique is based on the general architectural symmetry between normal right and left breasts, with asymmetries corresponding to possible masses. This method of identifying the locations of possible masses has been discussed in detail elsewhere ${ }^{4}$ and will be described only briefly in this article.

Initially the digital right and left mammograms are aligned using an automated procedure that has been described elsewhere. ${ }^{11} \mathrm{Next}$, the nonlinear bilateral-subtraction technique ${ }^{4,10}$ is performed for the initial identification of possible masses. The procedure includes generation of a set of gray-level threshold images from both right and left mammograms; bilateral subtraction between the corresponding right and left threshold images; and run-length linking of the multiple subtraction images. ${ }^{4,10}$ Figure 3 shows an example of run-length images for the right and left breast images, which were obtained from the original breast images shown in Fig 2. It should be noted that the mass is enhanced and the normal tissues are suppressed in the run-length images. The two runlength images are then thresholded to form binary images, which are subsequently processed $^{4}$ using a morphological closing opera- 


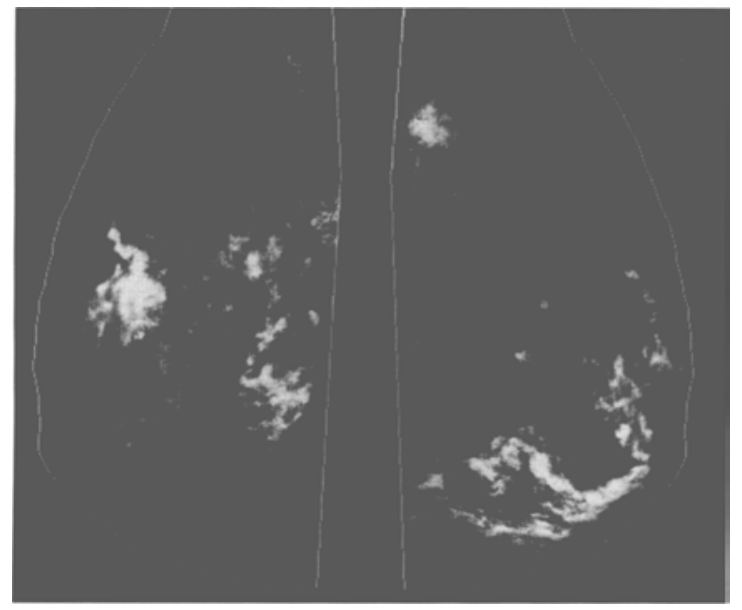

Fig 3. Example of run-length images for the right and lett breast images produced from the original breast images shown in Fig 1.

tion $^{12,13}$ and a morphological opening operation $^{12,13}$ to merge small neighboring regions (closing operation) and eliminate isolated pixels (opening operation), respectively. These resulting images are referred to as processed images in this article. Remaining regions suspicious for possible masses are then analyzed for the development of the feature-analysis techniques as described below to reduce the number of nonmass detections.

\section{Feature-Analysis Techniques}

Many of the suspicious regions initially identified as possible masses by the nonlinear bilateralsubtraction technique are not true breast masses. Mammographically, masses may be distinguished from normal tissues based on their density and geometric patterns as well as on asymmetries between the corresponding locations of right and left breast images. Not all of these features have been used in the initial identification of possible masses. Therefore, in a second stage of the mass-detection process, features such as the area, the contrast, and the geometric shape of each suspicious region are examined using various computer-based techniques, as will be described below, to reduce the number of nonmass detections (ie, to increase specificity). This analysis involves (1) the extraction of various features from suspicious regions in both the processed images and the original images, and (2) the determination of appropri- ate cutoff values for the extracted features in merging the individual feature measures to classify each suspicious region as mass or nonmass.

Features extracted in the processed images. The area of each suspicious region in the processed images is examined first to eliminate suspicious regions that are smaller than a predetermined cutoff area. The effective area of a mass in the digital mammogram corresponds to the projected area of the actual mass in the breast after it has been blurred by the $\mathrm{x}$-ray imaging system and the digitizer. The area of a suspicious region on the digital mammogram is defined as the number of pixels in the region, as illustrated by a solid-line contour in Fig 4. An appropriate minimum area might be determined on the basis of the effective areas of actual breast masses as outlined by a radiologist. However, because the area of each suspicious region identified by the computer in the processed image is dependent on the density information of that suspicious region and on the nature of the digital processing, the computer-

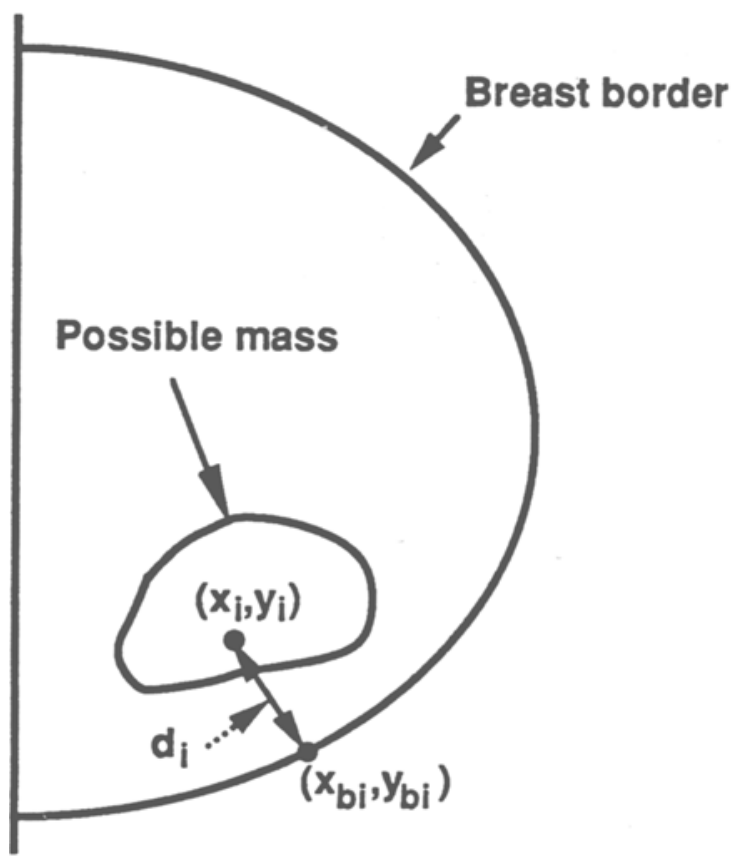

Fig 4. Schematic illustration of area and border-distance measures in the processed images. $\left(x_{i}, y_{i}\right)$ is one pixel location in the possible mass, and $\left(x_{b i}, y_{b i}\right)$ is the location in the border that is closest to $\left(x_{i}, y_{i}\right)$. Suspicious regions are obtained from the thresholded run-length images after morphological filtering operations. 
identified area may not be the same as the area in the original mammogram. Therefore, a method for the determination of the critical size cutoff for area discrimination will be introduced in a following section. This method is based on the distributions of areas of the regions that correspond to actual masses (actual-mass detections) and those that correspond to anatomic background only (nonmass detections).

A border-distance test was developed to eliminate artifacts arising from any border misalignment that occurred during the initial registration of the right and left breast images. Imperfect alignment produces artifacts that lie almost along the breast border in the processed image. In clinical practice, malignant masses are unusual in the immediate subcutaneous regions and are almost always clinically palpable. Therefore, for each computer-reported suspicious region, the distance, $d_{i}$, between each point inside the suspicious region, $\left(x_{i}, y_{i}\right)$, and the closest border point, $\left(x_{b i}, y_{b i}\right)$, is calculated as indicated in Fig 4. Distances calculated from all points in the suspicious region are then aver- aged to yield an average distance, $d$, as given by the following formula:

$$
d=\frac{1}{N} \sum_{i=1}^{N} d_{i}=\frac{1}{N} \sum_{i=1}^{N} \sqrt{\left(x_{b i}-x_{i}\right)^{2}+\left(y_{b i}-y_{i}\right)^{2}},
$$

where $N$ is the total number of points in the region. Notice that the location of $\left(x_{b i}, y_{b i}\right)$ varies with the location of $\left(x_{i}, y_{i}\right)$. If the average distance is less than a selected cutoff distance from the border, then the suspicious region is regarded as a misalignment artifact and is eliminated from the list of possible masses.

Features extracted in the original images. To examine the actual radiographic appearances of regions identified as suspicious for masses in the processed images, these sites are mapped to corresponding locations on the original digital mammogram. This involves automatically selecting a rectangular region in the original image based on the size of the suspicious region in the processed image. This rectangular region encompasses the suspicious region in the processed image, as shown in Fig 5. After the mapping, the
Fig 5. Mapping from the (A) processed image to the (B) original image. The region in the processed image is mapped to the original image by using a rectangular box. The suspicious (black) region in the original image is determined by a region-growing technique.
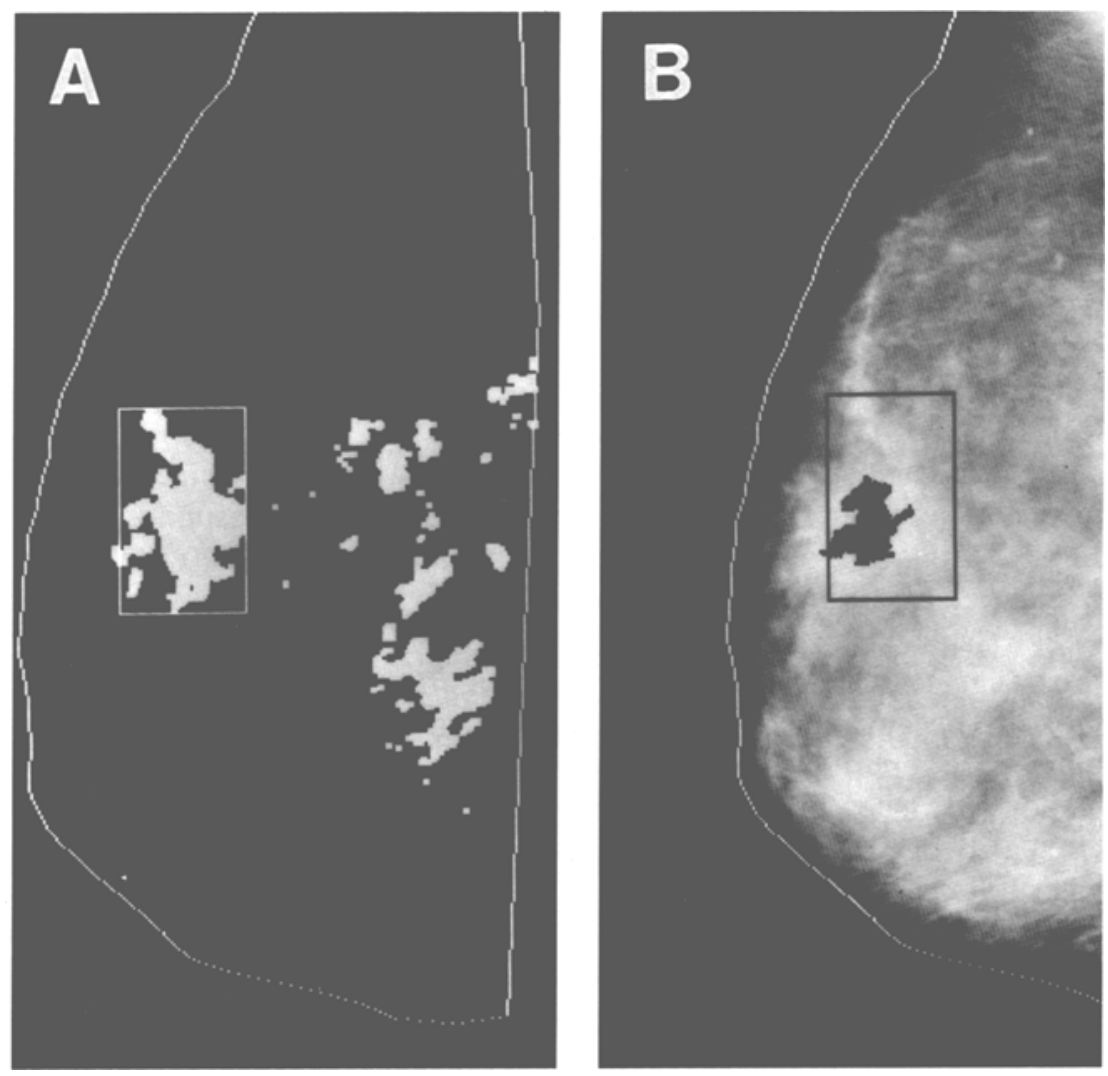
pixel location in the rectangular region having the maximum (peak) value is found. In this process, the image data are smoothed using a kernel of $3 \times 3$ pixels to avoid isolated spike artifacts when searching for the peak value. However, it should be noted that all following operations are performed on the original unsmoothed images. A corresponding suspicious region in the original image is then identified using region growing. ${ }^{14}$ In this study, the location with the peak gray level is used as the starting point for the growing of each region, which is terminated when the gray level of the grown region reaches a predetermined cutoff gray value. This predetermined gray-level cutoff was selected on the basis of an analysis of the 90 true masses on the original breast images. In this study, a cutoff value of $97 \%$ of the peak pixel value was selected empirically to prevent overestimating the area of true masses by the region-growing technique. However, it should be noted that this criterion tends to underestimate the area of many actual masses because of the strict cutoff value selected for region growing.

Each grown region in the original image is examined with respect to area, circularity, and contrast. The area of the grown region is calculated first. Similar to the area test used in the processed images, this test can be used to remove small regions that consist of only a few pixels. Because the cutoff value used in the region-growing technique in the original images is predetermined, the grown regions in the original images may become very large if the local contrast of the locations corresponding to suspicious regions identified in the processed images is very low. Therefore, some suspicious regions with an extremely low local contrast are eliminated by setting a large area cutoff.

The shape of the grown region in the original breast image is then examined by a circularity measure because the density patterns of actual masses are generally more circular than those of glandular tissues or ducts. To determine the circularity of a given region, an effective circle, whose area $\left(A_{c}\right)$ is equal to that of the grown region $(A)$, is centered about the corresponding centroid, as shown in Fig 6A. The circularity is defined as the ratio of the partial area of the grown region within the effective circle $\left(A_{p}\right)$ to the area of the grown region. The circularity test

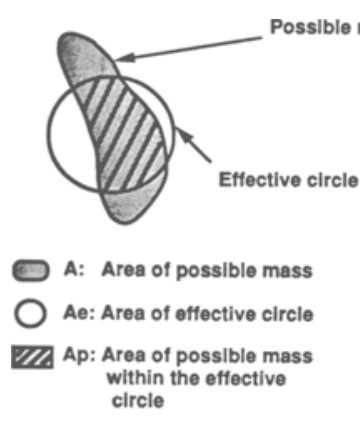

A

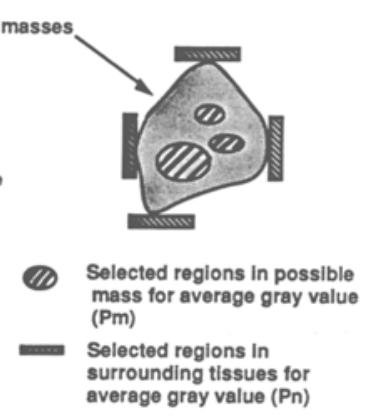

B
Fig 6. llilustration of (A) circularity and (B) contrast measures in the original images. The number of the pixels inside the circle is equal to the number of pixels in the grown region of a possible mass.

eliminates suspicious regions that are elongated in shape, ie, those having circularities below a certain cutoff value.

Actual masses are denser than surrounding normal tissues. A contrast test is used to extract this local density information in terms of a normalized contrast measure. Contrast can be defined as a difference in pixel values because the characteristic curve of the digitizer, which relates optical density to pixel value, is approximately linear. Contrast is defined here as the average gray-level difference between a selected portion inside the suspicious region $\left(P_{m}\right)$ and a selected portion $\left(P_{n}\right)$ immediately surrounding the suspicious region, as shown in Fig $6 \mathrm{~B}$. This definition of contrast is related to the local contrast of the mass in the breast image. In this study, $P_{m}$ corresponds to the average gray value calculated from pixels having gray values in the upper $20 \%$ of the gray-level histogram determined within the suspicious region and $P_{n}$ corresponds to the average gray value of four blocks (each $3 \times 9$ pixels in size) near the four extremes of the suspicious region (Fig 6B). Thus, the contrast test eliminates regions with low local contrast, ie, those that have similar gray levels inside and outside the suspicious region. In this study, a normalized contrast measure, defined as $\left(P_{m}-P_{n}\right) / P_{m}$, rather than contrast, defined as $\left(P_{m}-P_{n}\right)$, was used to characterize the contrast property of suspicious regions. It should be noted that the large area measure described above also provides contrast information of each suspicious region, which is mapped from the processed image and is subject to the area discrimination when the area of 
its corresponding grown region is obtained. However, the normalized contrast measure provides contrast information of each suspicious region, which is calculated from the grown region and is subject to the normalized contrast discrimination.

In the computerized detection scheme, various extracted features are merged sequentially in the order of area test (including areas in both the processed and original images), borderdistance test, circularity test, and contrast test (normalized contrast measure). The effect of varying this order will be discussed in a subsequent section.

\section{Optimization of Feature-Analysis Techniques}

The aim of using feature-analysis techniques is to reduce the number of nonmass detections without loss of actual-mass detections, that is, to increase specificity without loss in sensitivity. Therefore, optimization of the cuttoff values used in merging the values of the various features to distinguish between masses and nonmasses is an important step in the computerized detection scheme. In this study, the featureanalysis techniques are optimized by analyzing the cumulative histograms ${ }^{14}$ of both actual-mass detections and nonmass detections for each extracted feature. A cumulative histogram of a particular feature represents a monotonic relationship between cumulative frequency and the feature value. Here, feature value refers to the particular quantitative measure of the feature. For example, shape is characterized by a circularity measure that ranges from 0 to 1 . The corresponding cumulative histogram is calculated using the formula

$$
C(p)=\frac{p^{\prime}=\sum_{p^{\prime}=p_{\min }}^{p} F\left(p^{\prime}\right)}{\sum_{p^{\prime}=p_{\min }}^{p_{\max }} F\left(p^{\prime}\right)},
$$

where $p$ is a value of the extracted feature between the minimum value, $p_{\min }$, and the maximum value, $p_{\max }$, whereas $F\left(p^{\prime}\right)$ is the frequency of occurrence of the feature at the corresponding feature value $p$.

Cumulative histograms can be used to characterize each extracted feature and to determine an appropriate cutoff value for that feature. For each individual feature, cumulative histograms for both actual-mass detections and nonmass detections were calculated using the 308 mammograms (154 pairs with a total of 90 masses). Figures $7 \mathrm{~A}$ through $\mathrm{E}$ show cumulative histograms for the five features discussed earlier. These feature measures were calculated after nonlinear bilateral-subtraction. ${ }^{4}$ Figures $7 \mathrm{~A}$ and 7C show cumulative histograms for the area measures in both the processed images and original images, respectively. Note that, in Fig $7 \mathrm{C}$, an area of 30,000 pixels is used to represent those regions whose areas are equal to or greater than 30,000 pixels during the regiongrowing process in the original image. A very large area identified in the original image implies that the contrast of the corresponding suspicious region detected in the processed image is very low and, therefore, tends to be a nonmass detection. Figure $7 \mathrm{C}$ shows that a large proportion of the nonmass regions detected in the original images have large area. Figures 7B, $\mathrm{D}$, and $\mathrm{E}$ represent cumulative histograms for the border-distance, circularity, and normalized contrast measures, respectively. An example of the corresponding histogram for the circularity distributions is also shown in Fig 7D. It is apparent that cumulative frequencies of actualmass detections are lower than those of nonmass detections at small values for each of these extracted features. This indicates that more nonmass detections than actual-mass detections can be eliminated by setting a particular cutoff value for each feature. It should be noted also that it is possible to select cutoffs so that a certain percentage of nonmass detections will be eliminated while retaining all actual-mass detections. For example, as shown in Fig 7C, the nonmass detections can be reduced by $15 \%$ without loss of any actual-mass detections if a minimum area cutoff of 30 pixels is used in the original images. It should be noted that both minimum and maximum cutoffs can be used, as in the elimination of nonmass detections by use of the area test. This shows both the way in which the cumulative histograms can be used to select cutoff values of extracted features and the effectiveness of feature-analysis techniques in the reduction of nonmass detections. Based on the distributions of the cumulative frequencies and the requirement of high sensitivity, a set of cutoff values can be selected from the cumulative histograms such that no actual-mass detec- 


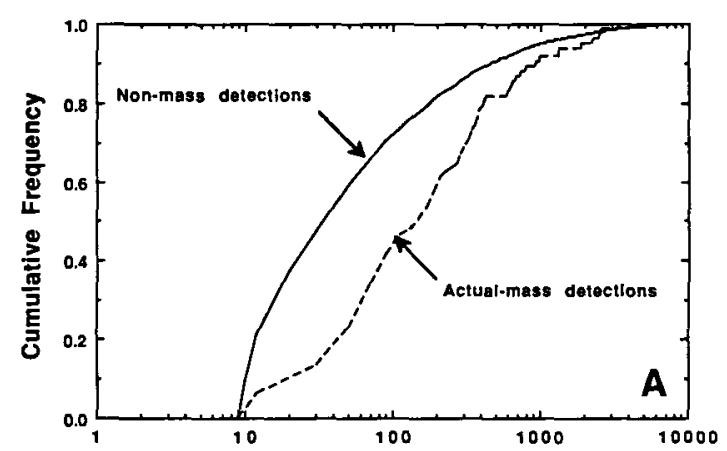

Area of Suspicious Region in Processed Image (pixels)

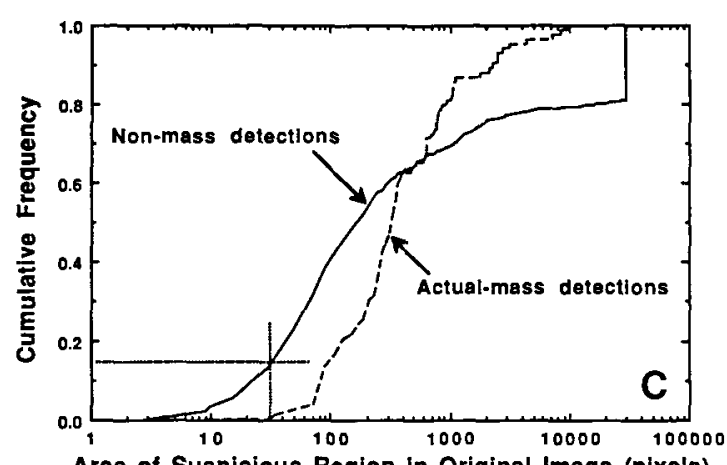

Area of Suspicious Region in Original Image (pixels)

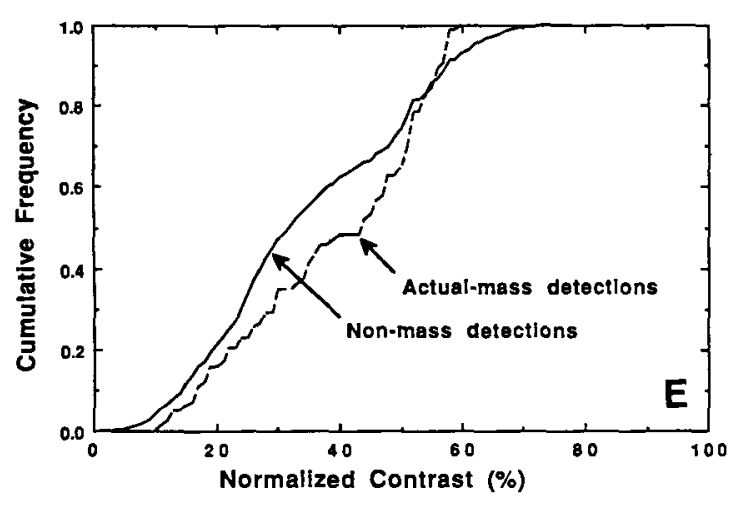

tions are eliminated, ie, such that sensitivity is not reduced.

\section{RESULTS}

The computerized scheme for the detection of possible masses was tested on the 154 pairs of mammograms in this study. Because the computerized scheme involves the multiple detection of possible mammographic masses in a single image, FROC analysis ${ }^{8,9}$ is used to evaluate detection performance. The performance data are fit to FROC curves using the FROCFIT program. ${ }^{8}$

Detection performance data were calculated for the computerized scheme that uses the
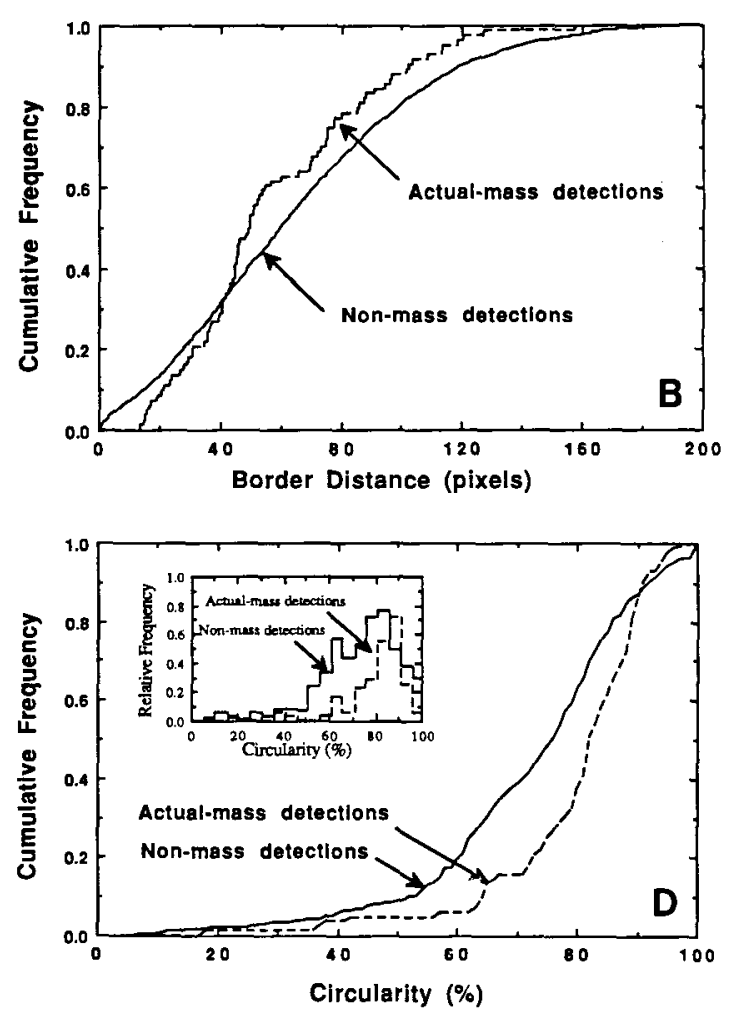

Fig 7. Examples of cumulative histograms of both actualmass detections and nonmass detections for $(A)$ the area and (B) the border-distance tests in the processed images; $(C)$ the area, $(D)$ the circularity, and $(E)$ the contrast tests in the original images. Data were generated from 154 pairs of mammograms. The inset in (D) shows the corresponding histogram.

nonlinear bilateral-subtraction technique for the initial identification of possible masses in combination with various feature tests. In this study, an area of 10,000 pixels (about $4 \mathrm{~cm} \times 4$ $\mathrm{cm}$ ) is always used as the cutoff for the large area measure, which corresponds to the area of the largest actual mass in the database. The cutoffs for all extracted features were set at levels such that no actual-mass detections were eliminated. It should be noted that these cutoff values are based on the particular set of clinical cases studied. Performance data consisted of sets of paired true-positive fractions, defined as the ratio of the number of actual masses de- 
tected to the total number of actual masses, and mean numbers of false-positive detections per image, defined as the ratio of the total number of nonmass detections to the total number of images. At each stage of feature testing, estimates of these points on an FROC curve were generated by varying the thresholding level that was used in the linking of the multiple-subtraction images. ${ }^{4}$ For example, at a sensitivity of approximately $80 \%$, the mean number of falsepositive detections per image is 3 . A total of five sets of performance data were generated, representing the performance of the computerized detection scheme before the use of feature tests, after addition of the area test, after addition of the border-distance test (ie, using both the area test and the border-distance test), after addition of the circularity test, and after addition of the contrast test. The detection scheme achieved a sensitivity of $91.1 \%$ at a false-positive rate of 9.9 per image before application of the feature tests and a sensitivity of $91.1 \%$ at a false-positive rate of 6.5 per image after application of all feature tests. Thus, the feature tests reduced the false-positive rate by $35 \%$ without loss of sensitivity.

Each set of performance data was fitted to an FROC curve as shown in Fig 8. The different curves in Fig 8 represent the performance obtained from the addition of the various stages of feature testing, starting with no feature tests and followed sequentially by the addition of the area, border-distance, circularity, and contrast

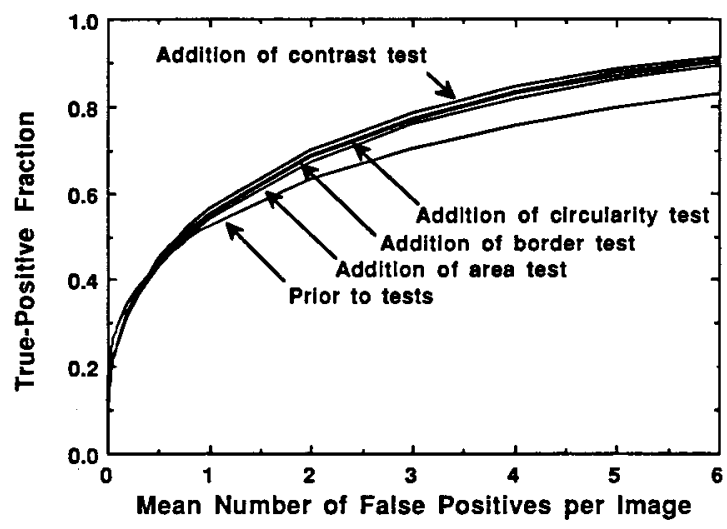

Fig 8. FROC curves generated at different stages of feature testing. These curves were generated using the nonlinear bilateral-subtraction technique at various threshold levels corresponding to different percent areas under the gray-level histogram of the subtraction images in the linking operation. tests. It is apparent that the feature tests improve detection performance substantially.

\section{DISCUSSION}

Figure 8 shows that when the area test is used first, its effect on the reduction of nonmass detections dominates that of other tests. For example, at a sensitivity of $91.1 \%$, the mean number of false-positive detections per image was reduced by $27.7 \%$ with the area test, an additional $2.5 \%$ by the border-distance test, $.5 \%$ by the circularity test, and $4 \%$ by the contrast test. About $41 \%$ of the nonmass eliminations achieved by the area test occurred because the area of the potential mass identified by the computer was large. The small effect of the border-distance test is related to gray level distributions in the breast images because gray values usually are lower near the skin line than elsewhere in the breast. The nonlinear bilateral-subtraction technique is based on the contrast distributions within single-breast images as well as between bilateral-breast images. Therefore, alignment artifacts may be suppressed because the pixels near the breast border may not have enough contrast to be identified (by thresholding) as suspicious regions. The small effect of the circularity test on detection improvement may be associated with the complexity of the density patterns of the suspicious masses that were extracted using the region-growing technique, which could yield small circularity values for some grown regions that correspond to actual-mass detections. However, the circularity test is expected to be improved when a better region-growing technique is developed for extracting suspicious regions in the original images.

In this study, the feature tests are implemented sequentially. Different sequences used in applying the feature tests may change intermediate results, but the final performance result must be the same when a given set of cutoff values is used because a suspicious region is classified as a mass if and only if it passes all of the individual feature test. The effects of all possible sequences of feature tests on detection performance was examined. By varying the number of feature tests included in all 24 possible sequences, the average numbers of nonmass detections per image eliminated by the addition of each feature in each sequence was 
determined. The overall number of nonmass detections eliminated by the full set of feature tests remained the same, regardless of sequence order, because the cutoff value for each feature was kept constant in all the different sequences. The area test eliminated many more nonmass detections than did the other tests. It was found that the amount of nonmass detections eliminated by each feature test varied slightly as the sequence changed. With sensitivity fixed at $91.1 \%$, the average percent reductions of nonmass detections by the area test, the borderdistance test, the circularity test, and the contrast test were $26.8 \%, 2.9 \%, .7 \%$, and $4.6 \%$, respectively. The corresponding standard deviations were $.3 \%, .3 \%, .1 \%$, and $.1 \%$, respectively, indicating that the variation in nonmass reduction caused by different sequences of the tests is negligible. It should also be pointed out that the feature measures could be merged alternatively in a parallel operation, such as that provided by an artificial neural network. ${ }^{15,16}$ This possibility will require further investigation.

\section{CONCLUSION}

To reduce nonmass (false-positive) detections, various feature-analysis technique were developed to extract various features of regions identified as suspicious for masses and to deter- mine appropriate cutoff values for the extracted features in merging the individual feature measures to classify each suspicious region as mass or nonmass. The features examined for each suspicious region involved the area in both the processed images and original images, the border distance in the processed images, and circularity and contrast in the original images. The appropriate cutoff values used for the various feature tests were chosen on the basis of cumulative histograms for both actual-mass detections and nonmass detections. In this study, the feature tests were merged in a sequential manner. The effectiveness of feature tests on the reduction of false-positive (nonmass) detections was evaluated using FROC analysis in combination with the computerized detection scheme that used the nonlinear bilateralsubtraction technique. Results showed that feature-analysis techniques were effective in the reduction of nonmass detections: approximately $35 \%$ of the nonmass detections were eliminated without loss of actual-mass detections (ie, without loss of sensitivity).

\section{ACKNOWLEDGMENT}

The authors are grateful to Dr C.E. Metz for his useful discussions and comments and to Dr Dev Chakraborty for providing his FROCFIT program.

\section{REFERENCES}

1. Feig SA: Decreased breast cancer mortality through mammographic screening: Results of clinical trails. Radiology 167:659-665, 1988

2. Tabar L, Fagerberg G, Duffy SW, et al: Update of the swedish two-county program of mammographic screening for breast cancer. Radiologic Clin North Am 30:187-210, 1992

3. Giger ML, Yin F-F, Doi K, et al: Investigation of methods for the computerized detection and analysis of mammographic masses. SPIE 1233:183-184, 1990

4. Yin F-F, Giger ML, Doi K, et al: Computerized detection of masses in digital mammograms: Analysis of bilateral-subtraction images. Med Phys 18:955-963, 1991

5. Tabar L, Dean PB: Teaching Atlas of Mammography, Stuttgart, Germany, Georg Thieme Verlag/Thieme Inc, 1985

6. Feig FA: Breast masses: Mammographic and sonographic evaluation. Radiologic Clin North Am 30:67-92, 1992

7. Sickles EA: Breast masses: Mammographic evaluation. Radiology 173:297-303, 1989

8. Chakraborty D: Maximum likelihood analysis of freeresponse receiver operating characteristic (FROC) data. Med Phys 16:561-568, 1989
9. Chakraborty D, Winter LHK: Free-response methodology: Alternate analysis and a new observer-performance experiment. Radiology 174:873-881, 1990

10. Yin F-F, Giger ML, Vyborny CJ, et al: Comparison of bilateral-subtraction and single-image processing techniques in the computerized detection of mammographic mass. Invest Radiol 28:473-481, 1993

11. Yin F-F, Giger ML, Doi K, et al: Automated registration of digital mammograms for use in mammographic computer vision schemes. Med Phys 18:524, 1990 (abstr)

12. Serra J: Image Analysis and Mathematical Morphology, Academic, New York, NY, 1982

13. Dougherty ER, Giardina CR: Image Processing-Continuous to Discrete, vol. 1. Englewood Cliffs, NJ, Prentice-Hall, $1987 \mathrm{ch} 3$.

14. Gonzales RC, Wintz P: Digital Image Processing (ed 2), New York, NY, Addison-Wesley 1987

15. Giger ML, Nishikawa RM, Doi K, et al: Development of a 'smart' workstation for use in mammography. Proc SPIE 1445:101-103, 1991

16. Wu Y, Giger ML, Doi K, et al: Artificial neural networks in mammography: Application to decision making in the diagnosis of breast cancer. Radiology 187:81-87, 1993 